\title{
THE SOCIOLOGICAL REALITY \\ OF THE HOMO INTERNETICUS
}

\author{
Bogdan Nadolu' ${ }^{1}$ (D) \\ Delia Nadolu ${ }^{2}$
}

DOI: https://doi.org/10.31410/LIMEN.2019.67

\begin{abstract}
The digitalisation of the everyday life has become a common reality for more than a half of the global population. To be connected 24/7 on several devices, to be at only one click/touch away from a huge amount of digital contents, to be available for interactions with almost any users from around the globe have become common facts. The insertion of the communication technology in our daily life is higher and deeper than never before. Into this paper, it was followed to identify the main sociological dimensions of the so-called Homo Interneticus - a new manifestation of the human being on the base of the new communication technologies. What are the main advantages of HI? But the disadvantages? Did we lose something from our human essence by the digitalisation of our existence?
\end{abstract}

Keywords: Internet, Smart-phone use, Online social presence.

\section{INTRODUCTION}

$\mathrm{D}$ uring the human evolution there where several events that had a massive impact over our beings: the biped walk, the development of the language, the tools, the agriculture, the printing. In the last almost half of century human civilization has known an unprecedented development of the communication technology, with direct and complex consequences for all dimensions of our lives (Castells 2009). The development and the spread of the New Information and Communication Technologies (NICT), under the global label of the Internet represent a massive social phenomenon that occurs from the 1980s on, with a quasi-anarchic unpredictable and uncontrolled evolution. Beginning as a secret military research project conducted by ARPA (Advanced Research Projects Agency) in 1969 the global network of computers, named firstly ARPANET and after 1990s Internet, has deployed a new era in our evolution: the information society (Luckett and Casey 2016). Inside of this unlimited digital universe anyone can say anything, anytime from anywhere (Doheny-Farina 1996).

More than half of global population access daily the digital universe because there are not any restrictions to going on-line, the proper technology is affordable and, last but not least the exploring of this new world is very enjoyable. The digitalisation of everyday life has become a common reality and, in many cases, even a necessity. To be connected full time from several devices simultaneous, to have at only one click/touch distance a huge amount of digital content, to can interact with any other user from anywhere have already become common facts. The communication technology is inserted in our life more extensive and more intensive than ever before. Our goals, values, opinions, behaviours, thinking, sociability, daily routine and social life have been significant reconfigured due the intensive use of the digital technology (Schaefer, Heinze, Rotte and Denke 2013).

\footnotetext{
West University of Timisoara, Bd.V.Parvan, no 4, Timisoara, Romania
}

West University of Timisoara, Bd.V.Parvan, no 4, Timisoara, Romania 
In 2004 Michael Goldhaber coined the expression Homo Interneticus as a "new form of human evolution based on their state of communication abilities". Even it can be considered mostly a philosophical one, we have to admit that it tends to become a very good descriptor of our daily status-quo. The Internet is an important part of human evolution: "... the invention of language was an evolutionary step of the human kind. The internet is a similar step" (Goldhaber 2019). Another reference to this formulation can be found in the Aleks Krotoski documentary movie "Homo Interneticus? - The Virtual Revolution" launched by BBC2 in 2012 (Krotoski 2012). The main issue of this film was how the web may be distracting and overloading our brains.

From a general point of view the Homo Interneticus is mostly defined with an excess of Internet use, so trend to have a negative connotation. Into a small research made in 2017 with an online questionnaire on an available sample $(\mathrm{N}=488)$ on this topic, over $70 \%$ of the subjects admit that we can nowadays talk about the Homo Interneticus as a new form of human being, and a similar percentage have declared that they don't want to become a Homo Interneticus. The main strengths of the HI where identified as the unlimited access to an enormous amount of information and the hyper-connectivity and the main weakness where the social alienation and the addiction by social media. So, into this light of these answers, to access intensively the Internet assure a high level of information and connectivity but, into the same time, generate alienation and addiction and thus, in the end it is a bad thing.

Of course, these are only subjective perceiving generated by the classical debate of the addiction issue, a subject that are coming from 1980s and was growing continuous until the Internet Addiction Disorder, officially recognized as a mental disorder. Even this aspect is as serious as possible, the intensive use of the Internet is not just a manner of addiction or psychological problem. Almost half of world population spent online at least two hours daily and this is just a social fact (Internet World Stats 2019). It is not necessary a debate about positive or negative consequences, it is happening and that is it. We can label ourselves as Homo Interneticus or not, the nowadays online social existence is a certitude. And this represent actually the main characteristic of the HI: to spend daily a minimal amount of time online. The question is how long? There is a clear milestone after we can talk about HI and before just a non-involved Internet user? For this, firstly we have made a very simply math following to calculate how many hours is necessary to generate the entire digital content that is quantified daily. For this we have started from the commercial statistics (Figure 1) that can be found on the Internet related to the idea "what's happening in an Internet minute?" (Desjardins, 2019). We have assumed that there is some inconsistency into this data but, they can be used at least as a general mark.

After that, we have established an estimated average time for each action (30 seconds / Google search, 3 seconds / WhatsApp message, 1 minute/ e-mail and so on) and have get the total amount of online time spend by the entire Internet population daily for generating the entire digital content and activities. And finally, we have divided for each activity the specific volume of time to their number of users (assuming that this is a very approximatively approach due to high dispersion between users). Thus, the final value was around 120 minutes. For generating all the online activity around the world is necessary than every user to spend daily 2 hours on him/her platforms and application (all on google search and email, but only some on Facebook, Twitter, Tik-Tok and so on). Even it is a low consistency of this value we can keep it only as a general milestone. We can assume, only as a hypothesis that the users that spend daily at least two hours online will be named HI and the others, with less than two hours daily can be named occasionally users or non-HI. Is this milestone functionally? Does have any relevance? 


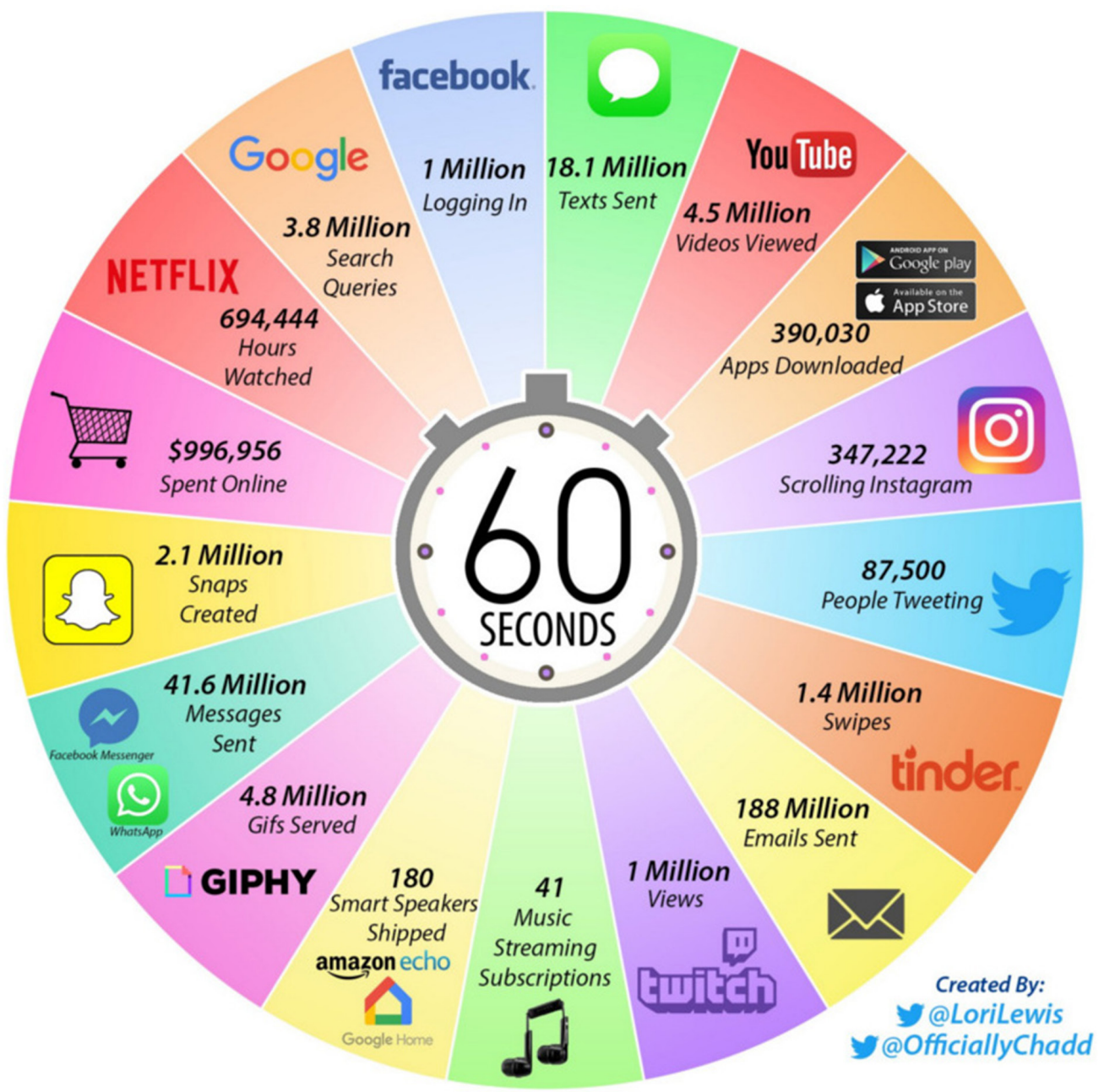

Figure 1. What is Happening in an Internet Minute in 2019

Sources: Jeff Desjardins, 2019

For this we have initiated an experimental survey with an online five items questionnaire, followed by Smartphone Addiction Scale - Short Version (Kwon, Kim, Cho and Yang 2013, Tudorel, Vintila, Vlaicu, Balauta, Goian and Rusu 2018) and ending with the request to upload a screen capture with the phone used during the last week ${ }^{3}$. This last part of the questionnaire was a real challenge, due to difficulties in obtaining the technical information from various type of smartphone and operating systems, but in the end, it was obtained 140 answers during May-July 2019. It was only a convenience sample, with subjects approached via Facebook using the snowball selections technique. There are not claimed of the representativeness, but the results can provide a useful point of departure for further work. The subjects were informed about the exclusively research purpose of the study and the anonymous and confidential profile.

The sample was formed mostly from young people (63\% below 20 years age), relatively balanced by gender $(61 \%$ women) that are accessing the Internet mostly form smartphone $(75 \%)$ but that are not addicted to smartphone (69\% non-addicted at SAS-SV score). Into the Figure 2 there are the most interesting factual data of this research:

For the iPhone users they have only to go into the setting menu and to make three screen captures with the screen time and the more frequent application. For non-iPhone users we asked them to install a small free application, UsageTime [Usage Time Application 2019] that provides immediately their phone utilisation for the last week. 

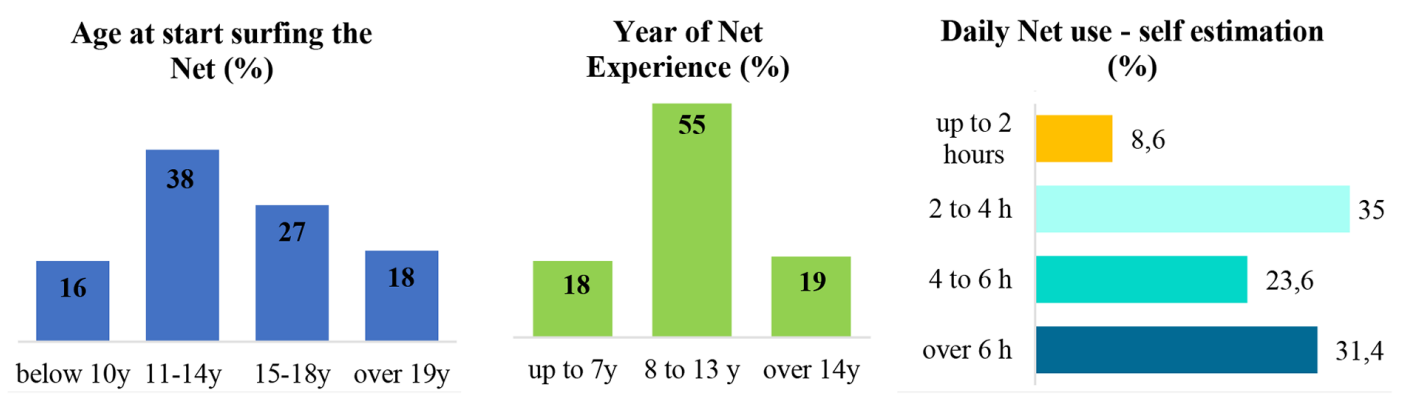

Figure 2 - Descriptive statistics of the factual distributions

Sources: Authors' work

On the base of previous milestone of two hours of online daily activities it was defined four categories of users: Non-Homo Interneticus (Non HI) - up to 2 hours, Light Homo Interneticus (Light HI) -2 to 4 hours, Medium Homo Interneticus (Medium HI) -4 to 6 hours and Strong Homo Interneticus (Strong HI) - over 6 hours. The main characteristics of each type of Homo Interneticus are presented in Table 1:

Table 1. Frequencies and characteristics of various type of Homo Interneticus

\begin{tabular}{|l|c|c|c|c|c|c|}
\hline $\begin{array}{l}\text { Self-estimation of the } \\
\text { time spends daily online }\end{array}$ & $\%$ & $\begin{array}{c}\text { Average } \\
\text { age }\end{array}$ & $\begin{array}{c}\text { Average } \\
\text { Net start }\end{array}$ & $\begin{array}{c}\text { Average } \\
\text { Mobile Use }\end{array}$ & $\begin{array}{c}\text { Average } \\
\text { peak } \\
\text { Mobile } \\
\text { Use }\end{array}$ & $\begin{array}{c}\text { SAS-SV } \\
\text { average } \\
\text { score }\end{array}$ \\
\hline up to 2 hours (Non HI) & 9 & $38 \mathrm{y} 9 \mathrm{~m}$ & $22 \mathrm{y} 1 \mathrm{~m}$ & $2: 14$ & $3: 21$ & 24 \\
\hline 2 to 4 hours (Light HI) & 35 & $27 \mathrm{y} 1 \mathrm{~m}$ & $16 \mathrm{y} 3 \mathrm{~m}$ & $4: 15$ & $6: 22$ & 28 \\
\hline 4 to 6 hours (Medium HI) & 24 & $25 \mathrm{y} 2 \mathrm{~m}$ & $14 \mathrm{y} 11 \mathrm{~m}$ & $4: 44$ & $7: 20$ & 30 \\
\hline over 6 (Strong HI) & 31 & $25 \mathrm{y} 1 \mathrm{~m}$ & $13 \mathrm{y} 11 \mathrm{~m}$ & $4: 39$ & $7: 16$ & 27 \\
\hline
\end{tabular}

There is a very clear and direct correlation between Internet use and age. All analyzed variables (the average age of starting use the internet, average daily mobile use and the average peak of mobile use) have a linear, direct but negative correlation with age. Also, it can be observed that if for Non-HI and for Light HI the daily online time is spent mostly from smart-phone for medium and strong HI the average mobile use remine below 5 hours, even the average peak goes over 7 hours. So, for a deep digital experience there are used multiple devices, not only the smartphone. Finally, one of the most important aspect is that all of these categories have obtained an SAS-SV average score below the cut-off point of addiction (31 for male and 33 for female). The Medium HI with four to six hours of online daily activities are the closest by the addiction point by smartphone. And this is also reflected into the highest level of mobile phone use (4 hours and 44 minutes) and the highest level of average peak ( 7 hours and 20 minutes).

\section{CONCLUSION}

Homo Interneticus, defined as an Internet user that spend daily more than two hours online, is a nowadays reality and is not based on the addiction to technology. The digital natives that are nowadays up to twenty years old have start to go online when they were teenagers and have a quite coherent behavior into the virtual space (socialization, entertainment, productivity). Even if they use almost five hours the mobile phone daily this is not a due to a clear addiction (the SAS-SV score was low) but to the alternative facilities offer by the mobile applications. The questions for the further research are: what will happen with our social space if people trend to reduce with almost five hours their offline availability? What will happen with the nowadays teenagers when they become adults? 


\section{REFERENCES}

Castells, M. 2009. Communication power. Oxford: Oxford University Press.

Desjardins, Jeff (2019) What Happens in an Internet Minute in 2019? available online at https:// www.visualcapitalist.com/what-happens-in-an-internet-minute-in-2019/

Doheny-Farina, S. 1996. The Wired Neighbourhood. Yale University Press.

Goldhaber, M.H. The mentality of Homo interneticus: Some Ongian postulates. Last modified 2004. Accessed February 24, 2019. https://firstmonday,org/ojs/index,php/fm/article/ view/1155

Homo Interneticus? The Virtual Revolution. 2012. Dir. Krotoski, A. BBC2. available at: https:// www.youtube.com/watch?v=jYpPt9d971I

Internet World Stats. Internet Usage Statistics. The Internet Big Picture. 2019. Accessed March 15, 2019. https://www.internetworldstats.com/stats.htm.

Kwon M., D.J. Kim, H. Cho. and S. Yang. 2013. The Smartphone Addiction Scale: Development and Validation of a Short Version for Adolescents. Plos One 8(12).

Luckett, O. and M. Casey. 2016. The Social Organism. New York: Hachette Book.

Schaefer M, H-J Heinze, M. Rotte and C. Denke. 2013. Communicative versus Strategic Rationality: Habermas Theory of Communicative Action and the Social Brain. PLoS ONE 8(5): e65111. https://doi.org/10.1371/journal.pone.0065111

Tudorel, O.I., M. Vintila, L. Vlaicu, D. Balauta, C. Goian and A. Rusu. 2018. Romanian Version of the Internet Addiction Test: Psychometric Properties and Cross-Gender Invariance. International Journal of Mental Health and Addiction. Springer Science+Business Media. DOI: $10.1007 / \mathrm{s} 11469-018-0014-6$

Usage Time Application software (version 1.4.4.). 2019. Vnova Mobile. 